This item was submitted to Loughborough's Research Repository by the author.

Items in Figshare are protected by copyright, with all rights reserved, unless otherwise indicated.

\title{
Healthy lifestyles to prevent dementia and reduce dementia symptoms
}

PLEASE CITE THE PUBLISHED VERSION

https://doi.org/10.1108/WWOP-12-2016-0036

PUBLISHER

Emerald

VERSION

AM (Accepted Manuscript)

PUBLISHER STATEMENT

This work is made available according to the conditions of the Creative Commons Attribution-NonCommercialNoDerivatives 4.0 International (CC BY-NC-ND 4.0) licence. Full details of this licence are available at: https://creativecommons.org/licenses/by-nc-nd/4.0/

\section{LICENCE}

CC BY-NC-ND 4.0

\section{REPOSITORY RECORD}

Hogervorst, Eef. 2019. "Healthy Lifestyles to Prevent Dementia and Reduce Dementia Symptoms". figshare. https://hdl.handle.net/2134/26528. 
Healthy lifestyles to prevent dementia and reduce dementia symptoms

Eef Hogervorst

Loughborough University

Bio:

Eef Hogervorst is Professor of Psychology and Head of the Dementia Research Group in the School of Sport, Exercise and Health Sciences at Loughborough University. She has published over 200 peer reviewed international publications on dementia and cognitive ageing and has obtained over $£ 9 \mathrm{M}$ with collaborators to fund her research in dementia

See: http://www.lboro.ac.uk/departments/ssehs/staff/eef-hogervorst/

\section{Correspondence}

Eef Hogervorst

Dementia research group

http://www.lboro.ac.uk/research/dementia/

School of Sport Exercise and Health Sciences

National Centre for Sports and Exercise Medicine

Loughborough University

e.hogervorst@lboro.ac.uk 


\begin{abstract}
Purpose Dementia is a growing problem worldwide, due to an ageing population. It has significant human and economic costs. There is no effective medical treatment.

Design/methodology Using earlier reviews, we discuss the difference between dementia and cognitive ageing and the different types of dementia, in terms of manifestation and pathology. We reviewed non-modifiable risk factors for dementia, such as age, gender, genetics and education. Education can possibly have lifelong protective effects, as it may promote cognitive reserve. This paper focused on lifestyle interventions to further increase brain reserve capacity. Findings from earlier reviews were summarised to provide guidelines for policymakers and practioners.
\end{abstract}

Findings Our reviews suggested that most nutritional approaches may have limited effectiveness, and should be implemented in midlife, before dementia symptoms are present and probably only in people who are actually nutritionally deficient. We found a reasonable positive evidence base of engagement in cognitive and physical activities to prevent dementia, which may also help reduce symptoms of dementia.

Practical and social implications From the studies reviewed, it was suggested that keeping physically and mentally active may help in all stages of life to prevent, but also reduce dementia symptoms. A focus on nutrition and treating heart disease risk factors is possibly limited to midlife, or before dementia symptoms are present.

\title{
Orginality
}

We used an evidence based approach to review which lifestyle interventions could help prevent dementia or reduce dementia symptoms. The main outcome of this paper was that stage of life and ability (dementia present or not) may interact with success of the intervention

Keywords: dementia, memory, activities, exercise, nutrition 


\section{Introduction: What is dementia}

Differences in brain function become larger with age, and not all cognitive functions age at the same rate within or between individuals. Cognitive function includes learning, memory and other forms of complex information processing, such as planning and inhibition, attention, recognition, language and visuospatial functions (e.g. used for reading maps). Morbidity (such as heart disease, diabetes mellitus and infectious disease) can worsen cognitive function and decline and is more common with age. While many of these often chronic diseases share risk factors with dementia, dementia is characterised by separate pathology, such as plaques and tangles seen in Alzheimer's disease, the most common type of dementia. So while dementia risk increases with age, it is not a normal effect of ageing and it needs to be distinguished from other illnesses which can also cause cognitive impairment.

The question is whether lifestyle changes can reduce dementia risk. We now know that what is good for the heart is good for the brain (Kivipelto, 2005; Hogervorst, 2012). This paper will describe protective lifestyles and dementia risk in more detail, but first discusses the most common different types of dementia, their symptoms and pathology and then non-modifiable risk factors for dementia.

\section{Types of dementia}

Dr Alois Alzheimer characterised Alzheimer's disease pathology in 'Uber Eine Eigenartige Erkrankung der Hirnrinde (on a peculiar disease of the cerebral cortex)' in 1907. He described the tangles and plaques found in the brains of people with this form of dementia, which was named after him. Clinically this type of dementia is usually characterized by an early loss of memory (problems in memory recall and learning of items, names, people and events), resulting in day-to-day forgetfulness, which is progressive and shows as a gradual but relentless loss of function. There should be other cognitive impairments as the disease progresses, such as visuospatial-; spatial and temporal orientation-; language-; and planning disorders and often personality change, which may become apparent early on in the disease in some cases. These changes should impact on social and/or occupational function and are not caused by other morbidity. For instance, thyroid disease is relatively common in older people and can cause memory dysfunction, but it can also usually be treated effectively. A clinical depression is seen in 1 in 5 people over 65 years of age and is often accompanied by memory problems, but this also responds to treatment relatively well. About $70-80 \%$ of people treated with antidepressant medication or cognitive behavioural therapy show improved mood and memory within 3 months. However, some people with AD will have a co-morbid depression and depression is thought to be a risk factor for dementia (Barnes \& Yaffe, 2011)

Vascular dementia is the next most common dementia, which progression is often more stepwise and which follows after strategic infarcts in the brain and/or extensive white matter disease in the brain (Hogervorst, 2003). Vascular dementia usually 
does not present with severe memory impairments first, as this tends to come later in the disease (Hogervorst, 2002b). Vascular dementia shows stepwise focal signs and symptoms (e.g. language impairments called aphasia and psychomotor problems called apraxia). Another common form of dementia is Lewy Body disease (where we find Lewy bodies in neurons which impair their function). It usually is seen with Parkinson's disease symptoms (e.g. the typical mask like face, problems in gait, a typical pill rolling tremor of the hands and depression). Lewy Body Disease is further characterized by visual hallucinations and confusion, and often language problems (word finding) come first, rather than memory impairments. Lastly, frontotemporal dementia usually first becomes apparent as personality change and issues with planning and inhibition. However, at post-mortem (which is when the diagnoses of dementia can only really be confirmed), we often see overlap of the dementia pathologies and it is not that common to see only one type of pathology (Hogervorst, 2003). Because there is no current effective long term treatment for any of these dementias, we need to focus on prevention.

\section{Risk factors we cannot change.}

Age and the female gender are risk factors for Alzheimer's disease (AD), the most common type of dementia. Women were possibly at a higher risk for AD type dementia (Launer, 1999), because they tended to reach an older age than men. An older age is a risk factor for $A D$, where at age 65 the risk is still low, this thereafter increases with each decade of life. As said, we now know that what is bad for the heart is bad for the brain and that includes increased AD risk. It is thought that healthy lifestyles traditionally thought to promote heart health (activity, a healthy diet with vegetables and fruit, not smoking) may offset some of this risk. However, possibly because more women are adapting unhealthy lifestyles (inactivity, smoking, alcohol abuse), these gender differences in risk for AD type dementia are now reduced in several cohorts (Hogervorst, 2012).

Another risk factor we cannot change is genetic. Whereas for the relatively rare early onset $A D$ (< age 65 years) there may be a stronger genetic link and we can often see this occur in first degree relatives, this is less clear for late onset AD. Here a genotype called APOE e4 (also associated with risk for heart disease) is thought to increase $A D$ risk. When you have one risk allele you double your risk, but if you carry both risk alleles (e4/e4, which is rare) your risk for AD might be 8 to 16 times higher. On the other hand, about $50 \%$ of AD cases do not carry the APOE e4 risk allele and about $20 \%$ of healthy controls carry one e4 allele but never develop AD (Hogervorst, 2002). This may be because of brain reserve capacity, which can act as a buffer.

Several papers (Whalley 2000; Hogervorst, 2013) described the positive relationship between childhood IQ and having obtained more education as protective and reducing dementia risk by extending the age of onset of dementia. This could reflect the effect of a positive childhood (or parental/maternal) experience with safe environments (e.g. less neurotoxicity in the environment due to pollution, good 
nutrition, adequate medical care provided, etc.) and/or access to stimulating environments (resulting in having more alternative information and intellectual coping when brain function starts to fail). According to the cognitive reserve theory (Esiri 2012;van Veluw, 2012), the amount of dementia pathology needs to override the initial brain reserve. So apart from education, how can we increase this reserve capacity?

\section{Risk factors we can change}

Lifestyle factors may be able to increase brain reserve capacity and reduce dementia risk, such as not smoking and no abuse of alcohol; increasing physical activity and reducing risk for head injury; and better nutrition (e.g. with sufficient micronutrients, such as folate, B12, and anti-oxidants).

\section{Heart disease risk factors}

Importantly, heart disease risk factors and nutritional deficiencies may be most effectively targeted in midlife to reduce later life dementia risk (Hogervorst, 2012). The age at onset of dementia is usually over 65 years. Whereas high blood pressure as a risk factor for dementia was seen 15 years before onset of the actual disease (in midlife), a decrease in blood pressure was shown 1-2 years before the onset of dementia (Skoog, 2003). At Oxford University, in the Oxford project To Investigate Memory and Ageing we also found that patients with $A D$ often have lower blood pressure than age matched controls (Hogervorst, 2002). This suggests that hypertension should be treated in midlife, at least two decades before the average age at onset of dementia. Similar effects were seen for total cholesterol levels and loss of body weight. For instance, in another Scandinavian study, midlife cholesterol levels per se were not associated with later life dementia (after 32 years of follow-up), but here investigators showed that the drop in cholesterol levels was the best predictor of subsequent onset of dementia (Mielke, 2010).

\section{Dental status}

It may be that blood pressure, cholesterol and weight all drop because the toxic plaques and other pathology affect those areas of the brain that are associated with metabolism and regulation of blood pressure, such as the autonomic nervous system. Another possibility is that these risk factors show a drop because people simply forget to eat, which is common in dementia. Because people eat less, they subsequently have a lower blood pressure (less weight, less salt intake) and cholesterol levels. Lastly, a lowered food intake could be associated with poor dental status. In Indonesia we found that elderly who had fewer than 10 teeth had a doubled risk for dementia (Hogervorst 2010). This association had also been found in the USA and Korea. Having fewer teeth could be the result of forgetting to brush your teeth and could result in a lower intake of essential nutrients to reduce dementia risk. A combination of factors (forgetfulness, dental status, etc) could of course also be possible. 


\section{Food intake: Vegetables, meat and B vitamins}

Having few teeth can interfere with food intake. This may be particularly true for hard to chew meat, whole grains, legumes, green leafy vegetables and nuts, which all contain B vitamins. Vitamin B12 has been associated with cognitive function and is difficult to obtain for people who do not eat meat or animal protein and only small levels are present in whole grains and potatoes. In China we found that both green vegetable and meat consumption had an independent positive association with memory function and a lower risk for dementia (Xu, 2014). Green vegetables are an important source of folate, which is also associated with cognitive function and memory. Legumes, such as lentils can also contain high levels of folate.

In the prospective Kungsholmen and several other studies following people over time, low folate or B12 status was associated with a doubled risk for dementia (see Smith, 2010). This may be because both folate and B12 can reduce levels of homocysteine. Homocysteine is a known risk factor for heart disease. In Oxford and other studies we found that high homocysteine was associated with markers of dementia in AD, such as deep white matter disease (Hogervorst, 2002); medial temporal lobe atrophy and cognitive function (Budge et al., 2003), but this was independent of other heart disease risk factors. Homocysteine can activate the cell cycle, meaning the neurons will try to divide, but end up dying. This could result in a trigger for formation of the plaques found in the brain. Homocysteine also sensitises neurons to excitotoxins and beta-amyloid toxicity resulting from the plaques.

Lowering homocysteine levels is possible. A meta-analysis of 12 randomised controlled trials found that only $0.5-5 \mathrm{mg}$ folic acid reduced homocysteine by $25 \%$ and $0.5 \mathrm{mg}$ B12 further reduced homocysteine levels by 7\%. Malouf et al (2003), however, showed in a Cochrane meta-analyses of 4 randomized controlled trials that there was no evidence for cognitive improvement in people with cognitive impairment after folic acid with or without vitamin B12/B6 treatment. In a later update of this meta-analyses (2008) with a further 4 studies ( 8 in total), they reported that there was some effect. A later study not included in this meta-analyses also showed a small global cognitive effect (vd Zwaluw, 2014) in older people who were folate deficient and who had been given folic acid, B12 and vitamin D for 2 years. However, these effects were not found on other cognitive domains. In an earlier Dutch study it was reported that in people with high homocysteine levels who had been given folic acid for 2-3 years, memory and information processing speed was also improved (Durga, 2007). A study in Oxford found that B vitamins reduced hippocampal loss by $30 \%$ in those with memory problems, but here these supplements had no effect on cognitive outcomes. The hippocampus is implicated in memory and dementia and its rate of decline is 10 times faster in people with dementia (Smith, 2010). 
Our meta-analyses (Lehert, 2015) showed no overall protective effect of B vitamin supplementation in treatment trials, but a trend was seen for improved global cognitive function and memory. This may be because B vitamin supplements only work for people who are folate deficient and/or have high homocysteine levels, but do not yet have symptoms of dementia, such as memory impairment. Including participants who are folate deficient and those who are not and for whom treatment may not be effective could affect analyses. Brain damage in dementia probably starts decades before the actually symptoms become apparent and early interventions should thus be recommended for those who are folate deficient. In the observational Health, Aging \& Body Composition we found low homocysteine levels after folic acid fortification had been introduced to flour in the USA. We analyses data of 3,075 white and African- American elderly aged between 70-79 years, with a 9 year follow-up to investigate the relationship between health conditions, body composition, social and behavioral factors. Of these people, 923 underwent cognitive tests and we also measured their bloods for homocysteine, folate, and vitamin B12. After fortification with folic acid, we found that nobody in this cohort had a deficiency in folate, nobody had homocysteine levels above the risk level (14 umol/L) and there was no (longer?) an association between homocysteine or the B vitamins and cognitive decline.

A recent meta-analyses mentioned in the New York Times showed no benefits of general vitamin supplements for health and effects of anti-oxidant or other vitamin supplements to prevent or treat dementia are much disputed. For instance, our meta-analyses showed no overall positive effect of vitamin $D$ supplements on global cognition or memory in older people (Lehert, 2015). It is also not clear if there is any benefit of vitamin A, C or E. It may be better to eat whole fruits and vegetables, whole grains and meats to provide $B$ vitamins and other essential vitamins.

\section{Food intake: Fats}

\section{Olive oil and the Mediterranean diet}

For instance, it may be the case that other compounds or combinations of foods are important in lowering risk for dementia and improving memory and global cognition. It was found that a combination of green leafy vegetables and olive oil resulted in an increased production of nitro fatty acids, which can lower blood pressure (by blocking epoxide hydrolase) (Charles, 2014). These types of salads are common in the Mediterranean where heart disease is lower than in the rest of Europe. Olive oil was hypothesized to also fight the amyloid plaques in the brains of people with Alzheimer's disease, because it contains a substance called oleocanthal. This substance is thought to affect many of the factors implicated in dementia. Infectious factors are increasingly thought to play an important role in dementia and olive oil was found to improve the immune system. Because olive oil/oleocanthal can also improve vascular health, there are thus several reasons why consuming olive oil could result in a reduced risk of dementia (Lorida, 2013). 
However, olive oil is used in the preparation of many Mediterranean dishes, which also potentially contain many other health promoting compounds. A review by Lorida (2013) showed that 9 of 12 studies (which were mainly observational) suggested that eating a Mediterranean diet was associated with better cognitive function, lower rates of cognitive decline and a reduced risk of $A D$. There have been a few treatment studies. One study showed that in more than 500 elderly who were at risk for heart disease, consuming the Mediterranean diet with added olive oil or nuts over a 6 year period (as compared to a low fat diet) gave people less risk of dementia (MartinezLapiscina, 2013). Our meta-analyses showed that adherence to the Mediterranean diet with nuts improved general cognition and that this diet with extra olive oil improved global cognition and verbal memory in older people. However, effect sizes were small (Lehert, 2015).

\section{Fish Consumption, Nuts and Omega 3 fats}

The Mediterranean diet is varied, but usually also contains fish. The Chicago Health and Aging Project showed that cognitive decline was $10 \%$ slower among subjects who consumed one fish meal per week and 13\% slower if they consumed two or more fish meals per week (Morris, 2003). Controls in this study consumed more fish rich in omega-3 fatty acids (i.e. anchovies, black pomfret) as compared to people with Mild Cognitive Impairment ( $\mathrm{MCl}$, who are at risk for dementia). Omega 3 fatty acids in fish are thought to be neuroprotective, can reduce vascular disease risk and non-hemorrhagic stroke risk. Omega 3 has antiarrhythmic, antithrombotic, antiinflammatory and antiatherogenic effects and reduces the synthesis of proinflammatory components, it can also maintain membrane integrity and neuronal function. Omega 3 fatty acids play a role in the expression of $B$-amyloid, a major component of the hallmark plaque pathology in AD. However, treatment studies using omega 3 have found contrasting effects and our meta-analyses showed no significant overall effect of omega 3 supplements on global cognition or memory (Lehert, 2015). Omega-3 is also found in nuts and our meta-analyses found a small protective global cognitive effect in people who ate the Mediterranean diet with nuts (Lehert, 2015). Again here whole foods rather than supplements may have more advantages, as other compounds could interact with one another in providing health promoting effects.

\section{Food intake: isoflavones}

\section{East Asian diets: Fruit and Soy products}

We found in Indonesia that eating fruit reduced risk of dementia and improved memory (Hogervorst, 2008). In this cohort, interestingly, we also found that consumption of green vegetables and tofu both had negative associations with memory and that both increased risk for dementia. For green vegetables this may be the case because use of pesticides is high in Indonesia which may have negative effects on brain function. For tofu this negative association with it consumption could 
be related to reports of its preservation in formaldehyde, which is neurotoxic (Hogervorst 2008). However, when we sampled tofu in Jakarta we could not find any evidence of this, but the practice may have been abolished after it became illegal. We also found similar negative associations of tofu and brain function in elderly in Hawai'i (Soni et al. 2016) and China (Xu 2014). In contrast, in women of middle-age we found that the phytoestrogens present in tofu protected against poor memory (Hogervorst 2011). Phytoestrogens, like estrogens, can protect the hippocampus against beta amyloid induced damage and have various other protective effects. However, (phyto)estrogens could have negative effects on brain function in older women (Hogervorst 2014). Treatment studies using soy or phytoestrogen supplements have shown variable effects (Soni, 2014) and our meta-analyses showed a very small positive effect of soy supplements on memory (Lehert, 2015), which was disputable and not clinically very relevant. As suggested, this may be related to differences in age of participants tested and the ethnic differences between studies (Soni, 2014), but could also be related to other foods in the diet of participants. We found associations in people of East Asian origin. In addition, tempe, a soy product that is fermented and contains B vitamins, such as folate and vitamin B12, was found to improve memory and reduce dementia risk and amyloid markers in both humans and animals (Kridawati, 2015, Soni, 2016, Hogervorst, 2010). It may be that folate off-sets potential negative effects of high levels of estrogens (Hogervorst, 2010)

\section{Activities: physical and cognitive stimulation}

Exercise can lower blood pressure, total cholesterol and improve cardiovascular function. It can reduce abdominal fat, which is thought to contain inflammatory cytokines (which were in Indonesia and other studies associated with worse cognition and dementia risk), it can improve lung and heart function, resulting in improved blood flow and better oxygen provision to the brain. It can also directly promote outgrowth of nerve cells needed for communication. In Indonesia and the UK we found that engaging in physical activity and sports reduced dementia risk and improved cognition and also that in those with dementia activity slowed memory decline. While a recent meta-analyses only including larger treatment studies found no overall effect of aerobic exercise on cognition, it did report a significant beneficial effect of t'ai chi in older people (Lehert, 2015). In contrast to this meta-analyses, our earlier reviews and those of others all showed significant beneficial effects of aerobic and strength exercise on cognition and physical ability in older without (Clifford, 2010) and with dementia (Hogervorst, 2012; McDermott, submitted). The latter review reported that three out of seven studies that included walking and four out of five studies that included getting up form a chair and walking a set distance (the timed get up and go test as an intervention) found beneficial effects on physical ability in dementia. One review mentioned optimal benefits of multicomponent exercise for a minimum of 12 weeks, 3 times a week for $45-60$ minutes. 
The majority of studies also found positive effects of physical activity on cognitive function in people with dementia.

In the latter review of reviews in humans also beneficial effects of cognitive stimulation therapy (but not cognitive training) were reported on brain function in people with dementia. Methodological differences between studies were significant and made it difficult to compare results and outcomes (Niederstrasser, 2016). A very early study in older rats found that an enriched environment increased connections between brain cells and it also resulted in more activity in rats (Diamond 1964). A large 4-arm 12 weeks treatment trial (Barnes et al., 2013) showed that the more older people with cognitive complaints engaged with whatever activity (mental or physical or even active control conditions), the better the outcome on cognitive function. Similarly, the FINGER study (Ngandu et al., 2015) also showed that combining physical and mental activities and reducing heart disease risk factors improved cognition and memory in older people at risk for dementia.

\section{Conclusions}

Risk factors for Alzheimer's disease are the same as those for heart disease, e.g. high blood pressure, smoking, high cholesterol, obesity, lack of folate/vit B12 (van Osch, 2004; Hogervorst 2002b, Kivipleto, 2005; Barnes \& Yaffe, 2011). Each of these factors about doubles the risk for dementia, but they act cumulatively (Kivipelto, 2005), so that if you have midlife high total cholesterol levels, body weight and blood pressure your risk for later life dementia could be increased by a factor 6 . According to Barnes and Yaffe (2011), by preventing diabetes, mid-life hypertension, mid-life obesity, smoking, depression, low educational attainment and physical inactivity, 10\% of AD cases worldwide and $25 \%$ in the US could be potentially prevented. Together, these risk factors are thought to contribute to up to half of AD cases globally (17.2 million). Several of these heart disease risk factors (obesity, smoking, high cholesterol/blood pressure, nutritional factors etc) probably need to be best treated in midlife to reduce the risk of dementia in later life.

So to reduce heart disease and dementia risk, in midlife at the latest, we need to stop smoking (it doubles the risk for dementia), brush our teeth, not eat too many sweets and visit the dentist regularly to keep our teeth healthy. This allows us to eat the good fats (nuts/fish/olive oil), a little meat, legumes (beans, lentils etc.), plenty of vegetables and fruits, and whole grains which is also important to do before dementia develops.

Before and when dementia has developed, conform governmental guidelines and reviews, we need to do aerobic exercises (to sweat, increase heart rate and improve lung function by walking briskly, or by going cycling or swimming for a total of 150 min per week) and also engage in muscle strengthening exercises (by using weights 2-3x/week, for 20-30 min or by heavy gardening (shovelling dirt) or carrying heavy 
shopping). Improving strength and balance will also reduce the risk for falls, which is increased in dementia and which itself increases risk for dementia. During exercise and in the heat especially it is very important to keep well hydrated. Adequate hydration is important as many older people lose their sense of thirst and often forget to drink. Hydration has also been found to improve cognitive function (Masento, 2014).

Depression was mentioned as a risk for dementia (Barnes and Yaffe, 2011) and in our current projects to treat dementia symptoms, we try to focus on promoting mental and physical activities which improve mood, often by including social aspects and promoting quality contacts ro reduce loneliness (Steptoe in prep, see also http://www.ucl.ac.uk/psychiatry/pride).

Future studies need to look at long term effects of improving cognitive reserve (possibly even through prenatal interventions) and this means that prevention of dementia requires a lifelong approach.

\section{Acknowledgements}

University of Indonesia (Prof Tri Budi Rahardjo); OPTIMA, University of Oxford (Professor A. D. Smith); MRC CFAS, University of Cambridge (Prof C. Brayne); PRIDE, UCL/Nottingham university (Prof Orrell). This work was supported with grants from the MRC, Wellcome Trust, RIA, NDA, ESRC/NIHR, ARUK, and the Newton fund/British Council

\section{References}

Barnes DE, Yaffe K (2011): The projected effect of risk factor reduction on Alzheimer's disease prevalence. Lancet Neurol, 10: 819-828. 10.1016/S14744422(11)70072-2.

Barnes DE, Santos-Modesitt W, Poelke G, Kramer AF, Castro C, Middleton LE, Yaffe K.(2013). The Mental Activity and eXercise (MAX) trial: a randomized controlled trial to enhance cognitive function in older adults. JAMA Intern Med. 2013 May 13;173(9):797-804. doi: 10.1001/jamainternmed.2013.189.

Budge, M., De Jaeger, C., Hogervorst, E., \& Smith, A.D. (2002). Total plasma homocysteine, age, systolic blood pressure and cognitive performance in the elderly. JAGS; 50;2014-2018 Charles et al (2014 Proc Natl Aca Sci).

Charles RL, Rudyk O, Prysyazhna O, Kamynina A, Yang J, Morisseau C, Hammock BD, Freeman BA, Eaton P. (2014) Protection from hypertension in mice by the Mediterranean diet is mediated by nitro fatty acid inhibition of soluble epoxide hydrolase. Proc Natl Acad Sci U S A. 2014 Jun 3;111(22):8167-72. doi: 10.1073/pnas.1402965111.

Clifford, A.C., Bandelow, S, Hogervorst, E (2010). Chapter 4. The Effects of Physical Exercise on Cognitive function in the Elderly. In the Handbook of Cognitive Aging: 
Causes, Processes and Mechanisms. Eds Quentin Gariépy and Raphaël Ménard Nova Sciences Publishers: NY.

Diamond M C, Krech D, Rosenzweig M R (1964)The effects of an Enriched Environment on the Rat Cerebral Cortex. J. Comp. Neurol. 123:111-119

Durga J, van Boxtel MP, Schouten EG, Kok FJ, Jolles J, Katan MB, Verhoef P. (2007) Effect of 3-year folic acid supplementation on cognitive function in older adults in the FACIT trial: a randomised, double blind, controlled trial. Lancet. Jan 20;369(9557):208-16.

Esiri MM, Chance SA (2012) Cognitive reserve, cortical plasticity and resistance to Alzheimer's disease. Alzheimers Res Ther. 2012 Mar 1;4(2):7. doi: 10.1186/alzrt105.

Hogervorst, E., Mendes Ribeiro, H., Molyneux, A., Budge, M., Smith A.D. (2002). Serum homocysteine, cerebrovascular risk factors and white matter low attenuation on CT scans in patients with post-mortem confirmed AD. Arch Neurology, May 59: 787-793.

Hogervorst, Eva, Marc Combrinck Pablo Lapuerta, Judith Rue Kate Swales Marc Budge (2002b). The Hopkins Verbal Learning Test and screening for dementia. Dement Geriatr Cogn Disord; 13:13-20

Hogervorst, E. Bandelow, S., Combrinck, M. Irani, S.. \& Smith, A.D. (2003). The validity and reliability of 6 sets of clinical criteria to classify Alzheimer's disease and vascular dementia in post-mortem confirmed cases: added value of a decision- tree approach. Dementia;16(3):170-180

Hogervorst, E, Sadjimin, T, Yesufu, A., Rahardjo, T. (2008). High tofu intake is associated with worse memory in Indonesian elderly men and women Dementia: 26:50-57

Hogervorst. E. Dementia and oral health. Invited keynote lecture Tokyo Japan Japanese Dentistry Society (JADS) October 22-24, 2010 http://kppikg.com/PDF/2.integrated-lectures.pdf

Hogervorst, E. Mursjid, F., Priandini, D., Setyawan, H., Ismail, R.I., Bandelow, S., Rahardjo, TB. (2011) Borobudur revisited. Invited paper Brain Research, epub Oct 28 2010, Mar 16;1379:206-12.

Hogervorst E, Clifford A, Stock J, Xin X, Bandelow S (2012) Exercise to Prevent Cognitive Decline and Alzheimer's disease: For Whom, When, What, and (most importantly) How Much?. J Alzheimers Dis Parkinsonism 2:e117.doi:10.4172/2161$0460.1000 \mathrm{e} 117$

Hogervorst, E., Clifford, A. (2013) What is the Relationship between Higher Obtained Education and a Delayed Age at Onset of Dementia. J Alzheimers Dis Parkinsonism 3, e128

Hogervorst, E. (2014) Oophorectomy and hysterectomy may increase dementia risk but only when performed prematurely. J Alzheimers Dis. 2014;42(2):583-6 
Kivipelto M, Ngandu T, Fratiglioni L, Viitanen M, Kåreholt I, Winblad B, Helkala EL, Tuomilehto J, Soininen H, Nissinen A. (2005) Obesity and vascular risk factors at midlife and the risk of dementia and Alzheimer disease. Arch Neurol. 2005 Oct;62(10):1556-60.

Kridawati, A Sulaeman, R Damanik, A Winarto, TB Rahardjo, E. Hogervorst (2016). Tempe Reversed Effects of Ovariectomy on Brain Function in Rats: Effects of Age and Type of Soy Product. The Journal of steroid biochemistry and molecular biology $160,37-42$

Martínez-Lapiscina EH ${ }^{1}$, Clavero P, Toledo E, Estruch R, Salas-Salvadó J, San Julián B, Sanchez-Tainta A, Ros E, Valls-Pedret C, Martinez-Gonzalez MÁ. (2013). J Neurol Neurosurg Psychiatry. 2013 Dec;84(12):1318-25. doi: 10.1136/jnnp-2012304792. Epub 2013 May 13.

Launer LJ, Andersen K, Dewey ME, Letenneur L, Ott A, et al. (1999) Rates and risk factors for dementia and Alzheimer's disease: results from EURODEM pooled analyses. EURODEM Incidence Research Group and Work Groups. European Studies of Dementia. Neurology 52: 78-84

Lehert, P., Villaseca, E Hogervorst, PM Maki, VW Henderson (2015) Individually modifiable risk factors to ameliorate cognitive aging: a systematic review and metaanalysis Climacteric, Oct;18(5):678-89

Lourida I, Soni M, Thompson-Coon J, Purandare N, Lang IA, Ukoumunne OC, Llewellyn DJ. (2013) Mediterranean diet, cognitive function, and dementia: a systematic review. Epidemiology. Jul;24(4):479-89. doi: 10.1097/EDE.0b013e3182944410.

Malouf R, Grimley Evans J.(2008) Folic acid with or without vitamin B12 for the prevention and treatment of healthy elderly and demented people. Cochrane Database Syst Rev. 2008 Oct 8;(4):CD004514. doi: 10.1002/ 14651858.CD004514.pub2. update from Cochrane Database Syst Rev. 2003;(4):CD004514.

Masento, N.A., Golightly, M., Field, D.T., Butler, .LT., van Reekum, C.M. (2014) Effects of hydration status on cognitive performance and mood. Br J Nutr. May 28;111(10):1841-52. doi: 10.1017/S0007114513004455. Epub 2014 Jan 30.

Mielke, M.M. et al (2010). The 32-year relationship between cholesterol and dementia from midlife to late life. Neurology. 75(21):1888-95

Morris MC, Evans DA, Bienias JL, Tangney CC, Bennett DA, Wilson RS, Aggarwal N, Schneider J. (2003) Consumption of fish and n-3 fatty acids and risk of incident Alzheimer disease. Arch Neurol. Jul;60(7):940-6.

NG Niederstrasser, E Hogervorst, E Giannouli, B Stephan (2016) Approaches to Cognitive Stimulation in the Prevention of Dementia Journal of Gerontology \& Geriatric Research online 3 July at: http://www.omicsgroup.org/journals/approaches- 
to-cognitive-stimulation-in-the-prevention-of-dementia-2167-7182-S5005.php?aid $=76480$

Ngandu T, Lehtisalo J, Solomon A, Levälahti E, Ahtiluoto S, Antikainen R, Bäckman L, Hänninen T, Jula A, Laatikainen T, Lindström J, Mangialasche F, Paajanen T, Pajala S, Peltonen M, Rauramaa R, Stigsdotter-Neely A, Strandberg T, Tuomilehto J, Soininen H, Kivipelto M. (2015) A 2 year multidomain intervention of diet, exercise, cognitive training, and vascular risk monitoring versus control to prevent cognitive decline in at-risk elderly people (FINGER): a randomised controlled trial. Lancet, 385, pp. 2255-2263

Skoog, I, Gustafson, D. (2003) Hypertension, hypertension-clustering factors and Alzheimer's disease. Neurology Sep;25(6):675-80.

Smith et al (2010) Homocysteine lowering trial ....accessed via http://journals.plos.org/plosone/article?id=10.1371/journal. pone.0012244

Soni M, Rahardjo TB, Soekardi R, Sulistyowati Y, Lestariningsih, Yesufu-Udechuku A, Irsan A, Hogervorst E. Phytoestrogens and cognitive function: a review. Maturitas. 2014 Mar;77(3):209-20.

Soni, M., White, L. R., Kridawati, A., Bandelow, S., \& Hogervorst, E. (2016). Phytoestrogen consumption and risk for cognitive decline and dementia: With consideration of thyroid status and other possible mediators. The Journal of steroid biochemistry and molecular biology 160, 67-77

Whalley, L. J., Starr, J. M., Athawes, R., et al (2000) Childhood mental ability and dementia. Neurology, 55, 1455 -1459

VanOsch, L., Hogervorst, E., Combrinck, M., Smith, A.D. (2004). Low thyroidstimulating hormone as an independent risk factor for Alzheimer disease. Neurology; Jun 8;62(11):1967-71

van Veluw SJ, Sawyer EK, Clover L, Cousijn H, De Jager C, Esiri MM, Chance SA. Prefrontal cortex cytoarchitecture in normal aging and Alzheimer's disease: a relationship with IQ. Brain Struct Funct. 2012 Oct;217(4):797-808. doi: 10.1007/s00429-012-0381-x.

van der Zwaluw NL, et al.(2014) Results of 2-year vitamin B treatment on cognitive performance: secondary data from an RC. Neurology. 2014 Dec 2;83(23):2158-66.

Xin Xu, Shifu Xiao, Tri Budi Rahardjo, Eef Hogervorst 2014 Tofu Intake is Associated with Poor Cognitive Performance among Community-Dwelling Elderly in China JAD, vol 43,no 2 